\title{
Gas Sensor Technology based on Titanium Oxide and Platinum-Carbon Layers
}

\author{
Mhamed El Achhab, E. Knorr, and Klaus Schierbaum \\ Department of Materials Science, Institute of Experimental Condensed Matter Physics \\ Heinrich Heine University, D-40225 Düsseldorf \\ mhamed.elachhab@hhu.de
}

\begin{abstract}
:
We report a sensor technology, based on plasma electrolytic oxidation, to form porous titanium oxide layers with well-defined phases and a reproducible microstructure on titanium substrates. By using a imprinting method to deposit graphite or catalytically active graphite-platinum electrodes, chemical sensors are obtained with high stability. Our design enables the application of a variety of different detection principles. They include non-isothermal and isothermal calorimetry, impedance spectroscopy as well as amperometry. Results are presented for $\mathrm{H}_{2} \mathrm{O}, \mathrm{H}_{2}, \mathrm{NO}_{2}$ and $\mathrm{CO}$ sensing with the most appropriate detection principle, electrode composition and titanium oxide microstructure.
\end{abstract}

Key words: PEO gas sensor technology, titanium dioxide, calorimetry, impedance, amperometry.

\section{Introduction}

Chemical sensors have key functions in various fields of application. We have developed a novel gas sensor technology with low power consumption $[1,2]$. The sensor elements consist of a plasma-electrochemically oxidized (PEO) titanium foil, coated with a graphite or catalytically active graphite-platinum top electrode. In the PEO process, titanium foils are immersed as anode in a suitable electrolyte; under the action of a large voltage various reactions take place at the oxide/electrolyte interface, including dissolution, electric breakdown, and plasma formation, eventually coupled with fusion of the metal [3]. The automatization of the plasma-electrolytic oxidation and an optimized procedure lead to excellent adhesion and stability of titanium oxide layers as well as reproducibility of the $\mathrm{TiO}_{2}$ chemical composition, crystal phase, and microstructure.

\section{Experimental}

Figure 1a) shows the laser-cut, oxidized and imprinted titanium foil before separation of the sensor elements. In the first step, titanium foil is laser-patterned. Subsequently, the PEO process is applied. Imprinting with a $\mathrm{C}$ or $\mathrm{Pt}: \mathrm{C}$ paste produces the sensitive top electrode. The microstructure of the $\mathrm{Pt}$ particles embedded in the graphite layer is revealed in figure $1 \mathrm{~b}$.
We have developed various digital, small-size electronic circuits for the sensors to realize various measuring principles of sensors, especially for humidity, hydrogen, carbon monoxide and nitrogen oxide, as well as a corresponding LabVIEW code to control and readout the electronics [2].

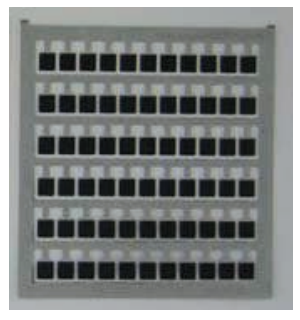

a)

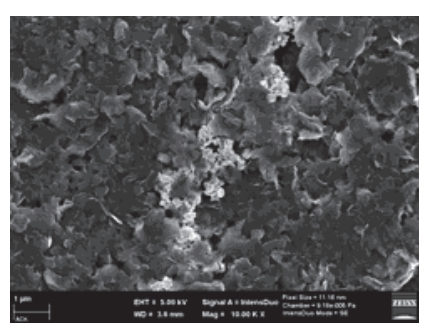

b)
Fig. 1. a) PEO sensors after printing the front electrodes. Laser cutting under argon is used to separate the individual sensor elements. b) SEM image of top electrode.

\section{Results and Discussion}

For hydrogen sensors with Pt:C layers, different detection modes can be used. By measuring the current under the action of a constant bias voltage, applied to the top electrode and the titanium substrate, hydrogen sensing is possible even in the absence of oxygen. This results from interfacial effects similar as observed for $\mathrm{Pt} / \mathrm{TiO}_{2}$ structures [4]. $\mathrm{H}_{2}$ in air below or at LEL concentration, can be also monitored with $\mathrm{Pt}: \mathrm{C} / \mathrm{PEO}-\mathrm{TiO}_{2} / \mathrm{Ti}$ devices that 
utilize catalytic turnover and the generated heat of oxidation. Two different electronic circuits are applied. The first type measures the temperature change with two thermistors in a "classical" Wheatstone bridge arrangement, one being covered with a $\mathrm{Pt}: \mathrm{C} / \mathrm{TiO}_{2} / \mathrm{Ti}$ foil [2] The second type uses a micro-heater, kept at a constant temperature of about $120{ }^{\circ} \mathrm{C}$ ("isothermal operation mode"). Here, the decrease of the electrical power $P$ serves as a measuring signal (Fig. 2). Both arrangements offer distinct advantages in terms of sensitivity and quantization as well as base-line stability in the presence of humidity.

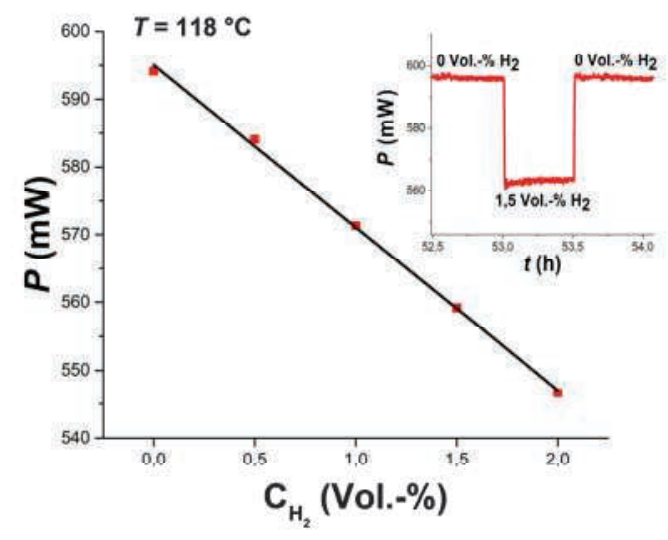

Fig. 2. Calibration curve of hydrogen sensor based on microcalorimetry. Inserted is a signal transient.

Sensors with a pure graphite top electrode can be used to measure the humidity in air. An impedance converter circuit is applied to determine the phase angle difference $\phi$ - $\phi_{0}$ with respect to the value in dry air (Fig. 3).

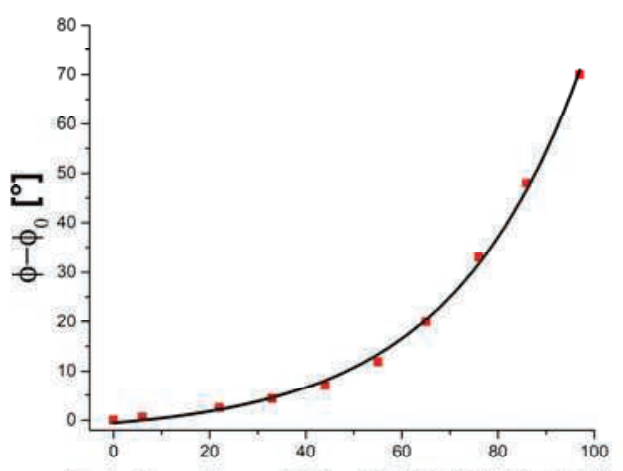

Relative humidity @ $23^{\circ} \mathrm{C}[\% \mathrm{RH}]$

Fig. 3. Calibration curve of humidity sensor with pure graphite top electrode.

The calibration curve of the sensor displays an exponential characteristics. The $\phi-\phi_{0}$ signals suggest a variable contribution of water dipoles and ionic conductivity as predominant detection mechanism.
We investigated the capability of PEO sensors for $\mathrm{CO}$ and $\mathrm{NO}_{2}$ detection at room temperature by means of an amperometric arrangement in which the conductivity of the $\mathrm{Pt}: \mathrm{C} / \mathrm{PEO}-\mathrm{TiO}_{2} / \mathrm{Ti}$ sensors is measured. We used a constant bias voltage, applied to the top electrode and the titanium substrate, and measured the current variations upon changes of the $\mathrm{CO}$ and $\mathrm{NO}_{2}$ concentrations in humid air. A typical result is shown in figure 4 . CO behaves like a donortype molecule. This effect requires a certain level of humidity in the air which suggests a more complicated interaction of $\mathrm{CO}$ on an atomistic scale. In contrast to $\mathrm{CO}$, the acceptortype $\mathrm{NO}_{2}$ decreases the conductivity of $\mathrm{Pt}: \mathrm{C} / \mathrm{PEO}-\mathrm{TiO}_{2} / \mathrm{Ti}$. Again, water has a noticeable cross-interference and its level in air must be constant to give an unequivocal reading of the $\mathrm{NO}_{2}$ concentration. Thus, even sub-ppm levels can be detected.

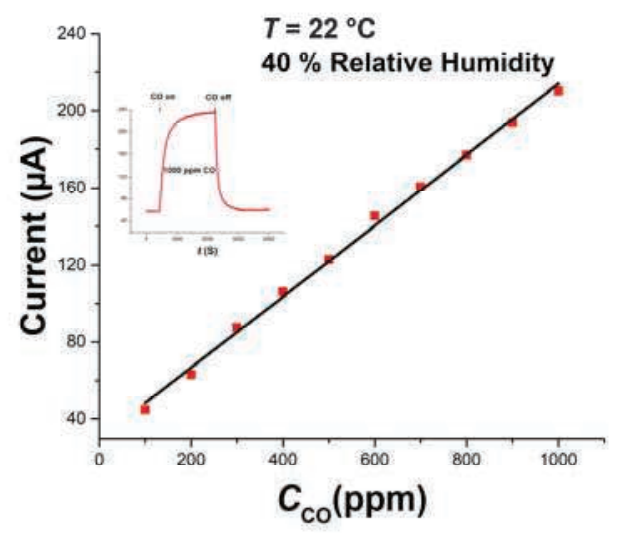

Fig. 4. Amperometric CO sensor at high-ppm $(0 . .1000$ ppm) concentration with Pt:C/PEO-TiO $2 / T i$.

\section{Conclusions}

The PEO gas sensor technology represents a platform technology for different gases and has the potential for comparatively low production costs. We demonstrated the applicability of different detection principles, including calorimetry for hydrogen, impedance measurements for humidity and amperometry for $\mathrm{CO}$ and $\mathrm{NO}_{2}$ detection.

\section{References}

[1] PTC/EP2012/070066 (submitted)

[2] M. El Achhab and K. Schierbaum, J. Sens. Sens. Syst., 5, 273-281 (2016); doi:10.5194/jsss-5-273-2016

[3] M. El Achhab, A. Erbe, G. Koschek, R. Hamouich, and K. Schierbaum, Appl. Phys. A, doi:10.1007/s00339-014-8392-5, 2014.A.

[4] A. Haidrya, A. Ebach-Stahl, B. Saruhana, Sensors and Actuators B 253, 1043-1054, (2017); doi: 10.1016/j.snb.2017.06.159 